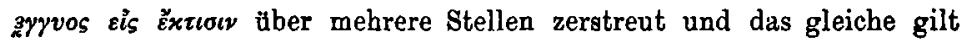
für die Ausführungen über die bürgschaftliche T'erminologie. Eine gedrängtere Systematik wäre daher vielleicht wirkungsvoller gewesen.

Doch sollen uns diese formellen Einwendungen die Freude an den schönen Ergebnissen des Buches nicht trüben. Der Verf., der seine Quellen suverän beherrscht, hat sich durch diese Arbeit in die erste Reihe derjenigen gestellt, welche sich die Erforschung des griechischen Rechts angelegen sein lassen, und die Universität Genf, zu deren 350 jährigem Jubiläum der Verf. sein Werk widmete, kann stolz sein auf diese Ehrengabe ihres Mitgliedes.

Prag.

Paul Koschaker.

Mélanges Gérardin, Paris, L. Larose \& L. Tenin, 1907. XV und 551 S. $8^{\circ}$.

(Fortsetzung von Bd. XXIX, S. 530.)

Der folgende Beitrag von R. Caillemer, der einzige, der nicht dem römischen Rechte angehört, bringt den Abdruck von 14 unedierten Klagschriften (libelli) aus dem Cod. Lat. der Bibl. Nation. 11724. Sie dienen, wie Verf. in der Einleitung ausführt, als Erläuterung zu den in derselben Handschrift enthaltenen Libelli iuris civilis et libelli iuris canonici des Roffredus; sie sind im 13. oder 14. Jahrhundert abgefaßt und stammen aus dem nordöstlichen Frankreich. Für die Kenntnis des französischen Rechts sind sie wichtig und wertvoll. In Nr. 3 dürfte auctentis in authenticis, nicht antedictis, aufzulösen sein; in $\mathrm{Nr} .10 \mathrm{Z.} 2$ ist zu lesen: ultro citro (die Hdsr. hat: ulto cit'), d. h. gegenseitig (invicem), geradeso wie hinc et inde auf der nächsten Zeile.

Der geistreiche Aufsatz von Collinet, der sich anschließt, behandelt die ursprüngliche Bedeutung der Stipulation (le rôle primitif de la stipulation). Sie diente ursprünglich lediglich dem Versprechen der Buße bei einem Delikt. Sie gab dem Verletzten einen klagbaren Anspruch, den er mit der Legis actio sacramento verfolgte. Eine Vertragsform, die alle Rechtsgeschäfte klagbar machte, war kein Bedürfnis der ältesten Zeit, das mit der Manzipation und dem Nexum völlig auskam und deren Rechtsleben unter dem Prinzip der Treue stand. Die älteste Sponsio war abstrakt in dem Sinne, wie sie das naive Empfinden des Urvolkes auffaßte, das nur auf die Haftung Wert legte, nicht auf ihren Grund, die spätere war gleichfalls abstrakt, aber infolge der verfeinerten Technik, welche konstruktiv die Verpflichtung von ihree Causa trennte. Diese Hypothese erklärt auch die Entstehung des Instituts der Bürgen. Bei der Manzipation waren sie entbebrlich, denn sie war Bargeschäft, beirn Nexum gleichfalls, denn hier baftete der Schuldner mit seiner Person; wer aber einen andern an Gesundheit oder Gut geschädigt hatte, wirde, auch wenn er versprochen hatte, den Schaden durch Geldbuße zu sühnen, vom Verletzten nicht ohne Bürgenstellung freigelassen. (Ganz ähnliche Gedanken entwickelt für. 
das griechische Bürgschaftsrecht Partsch.) Möglicherweise spondierten ursprünglich so gar nur die Bürgen, da ja sie allein sponsores heißen, nicht auch der BuBpllichtige. Das ist derselbe Gedanke, den kürzlich Mitteis (Festsch. f. Bekker S. 117 f., Romisches Privatrecht S. 269f.; dagegen Levy in dieser Zeitschrift XXVIII 401f.) ausgesprochen hat. Solche Wanderungen in das Nebelland der Orzeit sind stets ein anmutiges Spiel der Phantasie. Sie entbehren auch nicht des wissenschaftlichen Wertes; denn ohne Hypothesen ist es nicht möglich, die Anfänge der Institutionen aufzudecken. Begreiflich ist es auch, daß der Erfinder einer neuen Hypothese fest an sie glaubt und mit ihr den Schlüssel gefunden zu halen glaubt, der alle Schlösser öffnet. Es fragt sich nur, wie viele Gläubige er findet. Die Außenstehenden sind meist böse Skeptiker. Warum soll gerade beim Bußrersprechen die Sponsio nötig gewesen sein? Konnte nicht der Verletzte den Schuldigen in Haft behalten, bis ibn seine Familienangehörigen, seine Gentilen oder Freunde durch Zahlung der Buße befreiten? Oder war nicht hier das Nexum ebenso brauchbar, wie beim Gelddarlehn? Wer will das wissen!

Mit der folgenden Abhandlung kehren wir wieder auf historischen Boden zurïck. Cuq behandelt hier vortrefflich die i. J. 1906 zu El Ajustrel in Portugal gefundene Inschrift aus Hadrianischer Zejt, welche die Bergwerksordnung von Vipasca enthält (abgedruckt nach der ersten Publikation Cagnats von Mitteis in dieser Zeitschrift XXVII 356f., jetzt auch in der 7. Auflage von Bruns Fontes p. 293). Auch Mispoulet hat sie in verschiedenen Aufsätzen erörtert (Nouv. Rev. Histor. 1907 S. 1 f., S. 461 f., Revue générale du droit 1907, S. 20f.). Cagnat, Cuq und Mispoulet haben ihre Erklärungsversuche auch der Pariser Akademie vorgelegt. Sie weichen in wesentlichen Punkten stark voneinander $a b$, was sich hauptsächlich aus dem fragmentarischen Zustande der Inschrift erklärt. Anfang und Schlu $\beta$ fehlen, und gerade bezüglich der wichtigsten Punkte tappen wir im Dunkeln. Die neue Inschrift ist nach Cuq keine lex data, auch keine lex lata, sondern eine lex dicta, vergleichbar den afrikanischen Domänenordnungen, nicht speziell für das Bergwerk von Vipasca bestimmt, vielmehr ein Reglement für alle fiskalischen Silber- und Kupferbergwerke, eine loi-type, wie Mispoulet sagt, ein Normalstatut, das hier für ein bestimmtes Bergwerk zur Anwendung gebracht wird. Wie Cuq behauptet, kämen Silberminen für Vipasca ebensowenig in Betracht, wie der Abzugskanal (cuniculus qui aquam metallis subducet), von dem wiederholt die Rede ist. Die Inschrift trägt die Überschrift: Ulpio Aeliano suo salutum; der Anfang, der auf der vorhergehenden Tafel stand, fehlt. Wer war der Absender oder Verfasser? Nach Cuq der Minister der kaiserlichen Finanzen (procurator a rationibus), nach Mispoulet entweder der Statthalter Lusitanicus (legatus Augusti) oder der Prokurator dieser Provinz. Das Bergwerk von Vipasca gehört dem Fiskus als Domäne. Nach dem Rechte der früheren Kaiserzeit erstreckt sich das Grundeigentum auch auf die mineralischen Schätze unter der Erde und zwar unumschränkt; 
ein Bergregal gibt es nicht. In unserem Falle ist der Fiskus Grundeigentümer. Doch betreibt er den Bergbau nicht in eigener Regie; er überläßt die Ausbeutung der metallischen Schätze der Privattätigkeit und sichert sich nur einen Anteil am Gewinn. Die Natur und der Erwerb dieses Bergrechts nun sind es, welche der Erklärung die größten Schwierigkeiten bereiten und bezüglich deren die Ansichten der Erklärer am weitesten auseinandergehen. Da eine eingehendere Untersuchung hier nicht möglicb ist, so möge es mir gestattet sein, meine von Cuq allerdings stark abweichende Meinung mit allem Vorbehalt in der Kürze anzudeuten. Das Bergbaurecht wird erworben auf unbeschränkte Zeit, bedarf aber zu seiner Erhaltung der Ausübung; andernfalls erlischt es. Es ist veräußerlich durch lästige und wohltätige Rechtsgeschäfte unter Lelenden und von Todeswegen, allerdings mit gewissen Einschränkungen (Anzeigepflicht an den procurator metalli); es ist ferner teilbar und wird vielfach (vielleicht regelmäßig) genossenschaftlich ausgeübt. Unsere Inschrift bezeichnet es einmal als proprietas. Aber auf diesen Ausdruck ist ebensowenig Gewicht zu legen, wie darauf, daß das Erwerbsgeschäft als emptio venditio bezeichnet wird; denn die Inhaber des Rechtes beißen nicht emptores, sondern coloni. Das Wort proprietas darf nicht im strengen Sinn genommen werden. Es handelt sich vielmehr um ein dingliches Recht an fremder Sache, vergleichbar der Superficies und Emphyteuse, die auch Cuq heranzieht. Das Wort proprietas ist hier ebenso unpräzise gebraucht, wie bei unserm Bergwerkseigentum. Erworben wird es durch Occupatio, Adsignatio oder Emptio. Über die Assignation enthält die Inschrift keine Bestimmungen; sie wird aber im letzten Paragraphen elwähnt. Der Okknpation unterliegen teils bisher unbebaute Stellen, an denen, wie es scheint, jedermann das Schürfungsrecht hatte oder erwerben konnte, teils die rom früheren Erwerber verlassenen Schächte. Der Okkupant erwirbt aber niemals das Recht am ganzen Schachte, sondern nur an der Hälfte; die andere Hälfte verbleibt dem Fiskas, der in verschiedener Weise darüber verfügt (pars dimidia ad fiscum pertinens § 1; pars quae ad fiscum pertinet \$2; ut ex more pars dimidia fisco salva sit \$3; fälschlich bezieht Cuq die Worte auf die Bälfte der zutage gefórderten Erze; richtig Mispoulet). Die Analogie mit der Finderbälfte des Schatzes, die sich sofort aufdrängt, wird von Cuq hervorgehoben ond zugleich zutreffend daran erinnert, da $B$ Hadrian es war, der zuerst den Schatz zwischen Grundeigentümer und Finder teilte (J. 2, 1, 39. Cyylharz bei Glück S. 214). Der Fiskus kann den ganzen Schacht verkaufen (universum puteum proc. metallorum vendito $\$ 1$ ), demgemäß auch die ihm verbleibende Hälfte (ut ad eum pertineat proprietas partis quae ad fiscum pertinebit $\$ 2$, falls nicht non vor pertinebit einzuschieben ist). Er kann also den Okkupanten wider deren Willen einen Socius setzen, worauf sich vielleicht der $\$ 7$ bezieht, den Caq ganz anders deutet. Der Okkapant kann das nur verhüten, wenn es ihm gelingt, selbst dem Fiskus seine Hälfte abzukaufen. (Nach Mispoulet ist er dazu verpflichtet, aber von der Richtigkeit dieser Ansicht kann ich mich 
nicht überzeugen). Die Emptio findet statt in der Form der Versteigerung. Den Zuschlag erhält, wer das beste Gebot macht (qui primus pretium puteo fecerit); der Rechtserwerb aber hat bei Silberschächten zur Voraussetzung die Anzahlung von 4000 Sest. (et sestertia quattuor milia nummum fisco intulerit; ganz anders Cuq; wie hier, Mispoulet). Wie es bei Kupferminen gehalten wurde, wissen wir nicht. Zum Verkaufe kommen diejenigen Schächte, deren Inhaber Metalle geschmolzen haben vor Erlegung des Preises; ob auch andere und welche, ist nicht zu ermitteln. Läßt der Käufer sein Recht sechs Monate lang ungenutzt, so hat er es verwirkt; der Schacht wird dann frei für die Oklkupation. Der Okkupant verliert das Recht bereits durch zehntägige Arbeitspause. Wird der Bergbau genossenschaftlich betrieben, so hat jeder Genosse gegen die übrigen einen Anspruch auf Ersatz des entsprechenden Teils seiner Verwendungen, auch wenn diese nicht zugestimmt haben, ohne Rücksicht darauf, ob die Verwendungen notwendig oder nützlich waren. Dagegen muß die Kostenberechnung auf dem Forum drei Tage lang ausgestellt (propositum habeto) und auBerdem den ersatzpflichtigen Genossen durch den Praeco mitgeteilt werden (denuntiato). Erfolgt darauf keine Zahlung, so geht der Verpflichtete seines Anteils verlustig zugunsten des Ersatzberechtigten. Cuq, der alle diese Bestimmungen sehr schön erläutert, vergleicht dié bekannten römischen Bestimmungen über Hausreparaturen. Auffallend ist nun, daß der nächste Paragraph demjenigen, der gutgläubig Verwendungen gemacht hat, gegenüber den socii ein ius repetendi gibt. Über das Verhältnis dieses Paragraphen zum vorhergehenden bestehen wieder weitgehende Differenzen zwischen Cuq und Mispoulet. Doch muß ich es mir versagen, darüber zu bericbten; schon allzulange habe ich mich bei dem Aufsatze von Cuq aufgehalten, und ich verweise daher für den Rest die Leser auf die sehr beachtenswerten Ausführungen selbst. Angesichts dieser Abhandlung und der Tatsache, daß bereits drei französische Gelehrte über die neue Inschrift der Pariser A kademie Vorlesungen gehalten haben, während sie in Deutschland noch kaum beachtet worden ist, kann man sich eines Gefühls der Bitterkeit nicht erwehren. Unwillkürlich erinnert man sich der Bemerkung der Herausgeber dieser Zeitschrift im Jahrgang XXII, 195 und der leider nur allzuwahren Worte Lenels in dieser Zeitschrift XXIII, 100.

Declareuil, der nächste in der Reihe, schreibt über das römische Familienfideikommiß. Er zeigt, daß es erst im zweiten Jhdt. n. Chr. sich einbürgert, daß auf seine rechtliche Gestaltung namentlich Seaevola und Papinian Einfuß haben und daß es durch die Gesetzgebung der philosophischen Kaiser begünstigt und geregelt wird. Seine wichtigsten Erscheinungen sind das Fideicommissum post mortem und das fideikommissarische Veräußerungsverbot. Besonders verdienstlich ist die Ableitung des neuen Rechtsgedankens aus den Sitten und Anschauungen jener Zeit, mit denen Verf. sehr vertraut ist. Wir haben die Arbeit eines Rechtshistorikers vor uns, dem der Zusammenhang des Rechts mit dem 
Volksleben und der Volksseele keine leere These ist, der ihn vielmehr zum Ausgangspunkt und zur Grundlage seiner Forschung macht und dadurch schöne Früchte erntet.

Es folgt Deschamps mit seiner Abhandlung über den Ausdruck locare operas und die Arbeit als Vertragsobjekt in Rom. Er weist nach, da $B$ dieser Ausdruck von dem Vertrage, durch den ein Individuum sich verpflichtet, für einen anderen gegen Entgeld zu arbeiten, nie gebraucht worden wäre, wenn nicht die Zahl derjenigen, die über ihre Arbeitskraft verfügen konnten, in Rom sehr gering gewesen wäre infolge des Bestehens der Sklaverei, des Freigelassenstandes, der väterlichen Gewalt und der causa mancipii. Locare operas hat man zuerst bezüglich des Sklaven gesagt. Eigentlich. vermietete man nicht die Dienste des Sklaven, sondern den Sklaven selbst. Das war richtige Sachmiete. Freilich konnte nur der Eigentümer oder Eigenbesitzer die Sache selbst vermieten, nicht der Nießbraucher. Und als man erst die operae servi zum Gegenstand von Vermächtnissen machte, weil man gewissen Zweifeln und Schwierigkeiten, die sich aus dem NieBbrauchvermächtnis eines Sklaven ergeben konnten (partus ancillae), vorbeugen wollte, da war es gegeben, auch die Vermietung des Sklaven als locatio operarum servi zu bezeichnen. Derselbe Ausdruck wurde dann angewandt auf die Hauskinder, die Freigelassenen und die liberae personae in mancipio, bis man schließlich dahin gelangte, daß man auch dem homo sui iuris die Möglichkeit zugestand operas suas locare. Die römischen Juristen durchbrachen damit im kapitalistischen Interesse die Grundsätze ihres Rechts: dominus membrorum sworum nemo videtur und operae in resum natura non sunt. Dieser geistvolle Versuch, die locatio operarum zu erklären, verdient volle Beachtung neben dem Mommsenschen, der bekanntlich die Dienstverdingung aus der Apparitio des römischen Staats- und Verwaltungsrechts bergeleitet hat. Die Tendenz des Aufsatzes aber geht dahin, die 'atavistische' louage de services oder louage de travail aus dem französischen Recht zu verdrängen, und durch den contrat de travail zu ersetzen. Im B. G. B. ist dieses Ziel annähernd, wenn auch noch nicht vollkommen, erreicht.

Desserteaux sucht aus der Darstellung Cicero's pro Caecina c. 33. 34 zu erweisen, daß für die Römer ursprünglich die Begriffe civitas und libertas eine untrennbare Einheit bilden, da die eigentliche Freiheit nur besteht in dem 'liber esse ex iure Quiritium', was ohne civitas nicht möglich ist, daß demnach Verlust der civitas auch den der libertas zur Folge hat. Es sind Gedanken ähnlich denen, die Mommsen in seinem Aufsatz in der Festgabe für Beseler (Jur. Schr. III, If.) entwickelt hat, der aber dem Verf. unbekannt geblieben ist. Eine legis actio in iudicio (S. 186) war mir bisher noch nicht bekannt; was würde Wlassak dazu sagen?

Mit Scharfsinn und Energie sucht $D$ uquesne die Lenelsche Rekonstruktion der Cautio iudicatum solvi anzugreifen; er meint, sie sei der Cautio pro praede litis vindiciarum viel ähnlicher gewesen, als Lenel annimmt. In der clausula ob rem iudicatam sowohl wie in der 
clausula ob rem non defensam seien auf der Aktip- und Passivseite mehrere Personen aufgezählt gewesen, für und wider welche die Cautio Wirkung gehabt habe. Auch der Grund, aus welchem die Kautionsleistung auferlegt worden sei, sei in der Cautio selbst zum Ausdruck gebracht worden, wie aus Cicero's Quinctiana hervorgehe. Der Clausula ob rem iudicatam gibt Verf. folgende Fassung:

si ea res a Senpromio iudice quive in eius locum substitutus erit, secundum me heredem cognitorem procuratorem meum iudicata erit, quod. ob eam rem te heredem cognitorem tuum solvere oportebit.

Verf. stützt sich hierbei namentlich auf D. 50,16,158, wo auf Aktiv - und Passivseite der Stipulation der Erbe genannt ist. Lenel, der diese Stelle in seinem Edjkt und in der Palingenesie auf die Cautio p. p. l. $\nabla$. bezieht, hat bereits in einem Zusatz zur zweiten Auflage seines Edikts zu dem Aufsatze Duquesne's Stellung genommen und seine Resultate rundweg abgelehnt. Überzengend hat er ausgeführt, daß die Erwähnung des Prozeßvertreters auf aktiver wie passiver Seite unzweckmäBig gewesen wäre, und auch weder durch D. 3, 3, 27, 1 .noch durch D. 46,7,7 erwiesen wird. Darüber aber, ob das zitierte Fragment 50, 16, 158 auf die Cautio i. s. bezogen werden könne, hat er sich nicht geäußert. Vielleicht ist sein Schweigen nicht ganz bedeutungslos, so daß er bezüglich der Erwähnung des Erben Duquesne's Ausführungen wenigstens für beachtungswert hält, wenn er sich auch zu unbedingter Zustimmung noch nicht entschließen konnte.

Es folgt Esmein mit einem Aufsatz über die relative Wirkung der Rechtskraft (l'effet relatif de la chose jugké). Daß die In iure cessio des Nießbrauchs an einen anderen als den Eigentümer wirkungslos ist oder den Untergang des Rechts herbeiführt, daß die In iure cessio der erworbenen Erbschaft die Qualịtät des Erben nicht berührt, seine Forderungen vernichtet und seine Schulden bestehen läßt, daß die tutela cessicia, wenn sie weiter zediert wird, an den tutor legitimus zurückfällt, daß der im Miteigentum stehende Sklave, der von einem der mehreren Herrn freigelassen wird, nicht frei wird, sondern den übrigen anfällt, daß endlich der Nießbrauchsklave, der vom Eigentümer freigelassen wird, nicht die Freiheit erlangt, sondern servus sine domino wird, alle diese merkwürdigen Rechtssätze sucht Verf. daraus berzuleiten, daß ursprünglich die Rechtskraft des Urteils und der ihm gleichwertigen Confessio in iure im ScheinprozeB absolut gewirkt habe. Der Zedens habe sein Recht nicht nur gegenüber dem Zessionar verloren, sondern gegenüber jedermann. In einem Nachtrage verteidigt Verf. seine Hypothese gegen Rabels abweichende Auffassung (diese Zeitschr. XXVII, 309f.). In Zukunft wird er sich auch mit Wlassak auseinandersetzen mulssen, der erstens bestreitet, daß bei der In iure cessio eine Confessio stattfinde, und zweitens, daß die In iure cessio ein Scheinprozeß sei, da er den Prozeßbegriff für Rom auf ein rechtliches, durch Urteil zu lösendes Stre itverhältnis beschränkt (diese Zeitschr. XXVIII, 71f.). Inmerhin bleibt wertvoll und bemerkenswert an den Ausführungen Verfs., wie er den Übergang von der absoluten zur relativen 
Wirkung der Rechtskraft erklärt mit der Einsetzung des iudex privatus, von der er auch die Konsumptionswirkung der Litiskontestation herleitet. Sie war in streitigen Prozessen der letzte Akt, der sich vor der öffentlichen Autorität abspielte. Die Möglichkeit der Annäherung der Ansichten Verfs. und Wlassaks scheint uns danach nicht ausgeschlossen. Verf. nennt absolute Rechtkraftswirkung, was Wlassak für Ausfluß des magistratischen Imperiums erklärt. Im Grunde genommen komnt das auf dasselbe heraus.

Hinter und neben Ermein steht durch ein merkwürdiges Spiel des Zufalls sein "cher et éminent ami' Girard mit einem Aufsatze über die Geschworenen bei der Beleidigungsklage. Rekuperatoren haben nach ihm geurteilt in dem prätorischen Verfahren auf Grund der actio iniuriarum aestimatoria, nur wenn es sich um körperliche Mißhandlung handelte. Dieser Fall aber sei durch die lex Cornelia der prätorischen Iurisdictio entzogen und dem iudicium publicum überwiesen worden; daher hätte seit der lex Cornelia im iudicium privatum nur noch der iudex unus über Beleidigungen geurteilt. Dem steht entgegen, daß Gaius in den Institutionen (III 224) und Paulus in der Collatio 2, 6 auch die körperliche Mißhandlung im iudicium privatum und zwar vor dem iudex unus aburteilen lassen. Verf. sucht diese Schwierigkeit mit der Annahme zu beseitigen, daß Gaius, vielleicht auch Paulus, über Provinzialrecht schreibt, wo die Quaestionen der leges Corneliae nicht zur Anwendung kamen, und daß Paulus nach einem Erlaß des Severus oder Caracalla schrieb, der die Bestimmung der lex Cornelia über die Gerichtskompetenz bei Beleidigungen beseitigte. Aber Gajus spricht fortwährend vom Prätor und nicht vom Statthalter, und der Inbalt jenes kaiserlichen Erlasses ist uns nur ganz mangelhaft bekannt. Nach Dig. 47, 10, 7, 6 (wo das sed et sinnlos ist, es müßte mindestens scilicet et heißen) müßte man annehmen, daß nur die iniuria atrox durch die lex Cornelia rom Privatrerfahren ausgeschlossen worden sei. Aus dem Worte 'iudex' an den Digestenstellen, welche von der Beleidigung handeln, ist nichts zu entnehmen. Es kann überall interpoliert sein, und auch wenn es das nicht ist, so hat es eine allgemeine Bedentung, wie Verf. selbst zugeben muß. Auch Lenel in den Zusätzen zur zweiten Auflage des Edikts hält Girards Hypothese nicht für bewiesen.

Von der Natur der Dos handelt Gradenwitz im folgenden Aufsatz. Er zeigt, daß die Auffassung der Dos als Beitrag za den Lasten der Ehe spätklassisch ist und zuerst bei Papinian begegnet, daß der die Dos empfangende Ehemann kein Käufer ist, wohl aber in gewissem Sinne ein Gläubiger, dessen Vertrauen auf die Gültigkeit der Bestellung nicht enttäuscht (decipi, was durch Heranziehung von BGB. $\$ 307$ sehr fein erläutert wird) werden darf, dessen Erwerbsgrund, wenigstens in Konkurrenz mit einer causa lucrativa, nicht als lukrativ angesehen wird, und er verwendet djese Ergebnisse zur Erklärung des Pap. Oxy. 496, eines sorgfältig redigierten, alle Details genau regelnden Ehevertrages, in dem gleichwobl dem Ehemann kein Eviktionsanspruch bezüglich der Dotalsachen gewährt wird. Dieses Schweigen über einen Punkt, 
über den die Kaufpapyri so viel Worte machen, hält Verf. für bedeutungsvoll; er findet darin eine Übereinstimmung mit dem weströmischen Dotalrecht.

In einem durch scharfsinnige Exegese vieler Quellenstellen ausgezeichneten Aufsatze erklärt Herzen Dig. 20,3,3. Es handelt sich nach $\mathrm{ibm}$ in diesem vielbesprochenen Fragmente um die Abfindung eines Pfandgläubigers durch einen Dritten, nicht durch einen zweiten Hypothekar, und zwar deshalb, weil zur Zeit des Titius Aristo, von dem das Fragment zu seinem größten Teile herstammt, Bestellung mehrerer Hypotheken von verschiedenem Range an derselben Sache dem römischen Rechte noch unbekannt waren. Wenn eine Sache zum zweiten Male verpfändet wurde, so erlangte die zweite Verpfändung nur unter der Bedingung Gültigkeit, daß das erste Pfand getilgt wurde. In dem Fragment 3 scheidet Verf. als dem Paulus gehörig aus die Sätze: quo casu - efficietur, non per oblivionem - vendere, quod admittendum - habere potest. Auch der Satz neque enim - de pignore wird mit Manigk dem Paulus zuzuweisen sein. Der vom Verf. vermißte Beweisgrund dafür scheint mir in dem persönlichen Gebrauch von convenire zu liegen; vgl. Voc. Iurispr. Rom. I p. 1018, $22 \mathrm{f}$.

Im folgenden Aufsatze behandelt $\mathrm{H}$ uvelin die schwierige Stelle Cic. de orat. I, 36, 166-167. Dort wird von einem Prozeß gegen einen treulosen Vormund berichtet. Die Klage ist vermutlich die Actio de rationibus distrahendis; der Kläger macht in iure eine Forderung geltend, mit der er wegen pluris petitio vor dem Iudex sachfällig werden (causa cadere) muß. Der Beklagte wehrt sich dagegen, anstatt sich, wie es sein wahrer Vorteil erheischte, auf diese Klage einzulassen. Das Verfahren wird ausdrücklich als legis actio bezeichnet (quod alter plus lege agendo petebat). Verf. glaubt, daß es sich um legis actio per iudicis postulationem handele. Die Schuldfrage sei schon in der legis actio sacramento zuungunsten des Beklagten entschieden. Die legis actio per iudicis postulationem diene hier, wie in vielen anderen Fällen, als Nachverfahren zur Absehätzung der Kondemnationssumme. Aus der legis actio p. i. p. in dieser Funktion habe sich der Formularprozeß mit der Geldkondemnation entwickelt. - Ich bedaure, diesmal dem scharfsinnigen Verfasser nicht zustimmen zu können. Ich bestreite, daß turpe tutelae iudicium bedeuten kann: das schimpfliche Urteil in Vormundschaftssachen. Ich behaupte - Beweis vorbebalten - daß im ZivilprozeB iudicium niemals Urteil heißt. Verf. beruft sich zu Unrecht auf mich; meine Ansicht habe ich ausführlich entwickelt an einer vom Verf. nicht beachteten Stelle meines von ihm mehrfach zitierten Aufsatzes (diese Zeitschr. XVI S. 165), und ich fasse sie jetzt noch schärfer, als vor 13 Jahren, in den eben ausgesprochenen Satz zusammen. Auch an keiner der Stellen, die Verf. S. 349 n. 2 zusammengetragen hat, beißt iudicium 'Urteil', mit Ausnahme von Plaut. Curc. 5, 3, 39; aber diese Dichterstelle bat nicht die geringste Beweiskraft. Selbst zugegeben, iudicium heiße wirklich bisweilen 'Urteil', so kann das höchstens dann der Fall sein, wenn es allein steht, nicht aber, wenn 
es, wie hier, mit dem Genitiv verbunden ist, der die Art der Klage bezeichnet. Sodann können die Worte calter iniquum putabat plus secum agi, quam quod esset in actione sich nicht auf ein vorangegangenes Verfahren, sondern nur auf das schwebende beziehen. Überall, wo uns ron einem Schätzungsverfahren in römischen Proze $\beta$ berichtet wird, urteilt derselbe oder dieselben Richter, welche über die Schuldfrage entschieden haben. Es ist undenkbar, daB nach Entscheidung der Schuldfrage erst wieder in iure rerhandelt werden muß, damit das Schätzungsverfahren eingeleitet werde. Es ist endlich zum mindesten sehr unwahrscheinlich, daß der Kläger im Arbitrium de lite aestimanda noch sachfällig werden konnte. Verf. sagt selber, Absolution gab es hier nicht mehr, und er hilft sich mit der Annahme, daß der Richter die Verurteilung unterlassen durfte. Er unterscheidet zwischen absolvere und non condemnare. Aber diese Lösung ist höchst problewatisch trotz der feinen Beobachtungen, die Verf. bei dieser Gelegenbeit macht. Und hätte wirklich der Richter im Schätzungsverfahren den Kläger wegen pluris petitio abgewiesen oder abweisen können, so hätte er darum doch nicht den Beklagten von der Infamie befreit, die vielmehr durch die Verurteilung im Hauptrerfahren schon herbeigefuibrt sein nußte. Sejen wir doch ehrlich! Was wissen wir über die l. a. per iudicis postulationem? Nichts weiter, als die 7 Worte beim Probus. Darauf ganze Gebäude errichten zu wollen, ist aussichtslos. Ich halte es nicht einmal für ausgeschlossen, daB das lege agendo in Ciceros Erklärung nicht wörtlich zu nehmen ist, sondern einfach das Verfahren in iure bezeichnet. Daß übrigens Buvelins Aufsatz außerordentlich belehrend und anregend ist, bedarf wohl einem so ausgezeichneten Verf. gegenüber keiner besonderen Hervorhebung.

Der folgende Aufsatz ron Iobbé-Duval, Explication de la loi 16 au code 'de inofficioso testamento' 3, 28 hat die Inofiziositätsquerel zum Gegenstande. Verf. weist darauf hin, daß, wie schon Stephanus in den Basilikenscholien 39, 1, 1 auseinandergesetzt und nach ibm Viglius Zuichemus betont habe, die Ausdrücke agere de inofficioso testamento, actio de inofficioso testamento, actio inofficiosi testamenti nicht von einer besonderen Klage (oder Actio) zu verstehen sein. Sie bezogen sich auf ein Inzidentverfahren in iudicio. Die Zulässigkeit dieser Ausdrucksw eise sucht Verf. za erweisen. Er begegnet sich in seinen Ausführungen mehrfuch mit Hans Hellwig, Erbrechtsfeststellung und Reszission des Erbschaftserwerbes, Leipzig 1908, über dessen Ergebnisse er aber weit hinausgebt, indem er alle Fälle der Querela dem Inzidentrerfahren zuweist. Dieses wurde nach ihm durch ein Bew eisinterlokut eröffnet und durch eine Pronuntiatio geschlossen. Geschaffen war es durch die Zentumvirn, nachgeahmt aber wurde es auch vom iudex unus und in der cognitio extraordinaria. Zuständig waren für die hereditatis petitio in Rom, wenn es sich um den Nachlaß eines römischen Bürgers handelte, ausschließlich die Zentumvirn. Prätorisehe Erben erbaten die bonorum passessio litis ordinandae gratia, auf Grund deren sie nicbt das Interdictum quorum bonorum erhielten, wobl aber die petitio hereditats possessoria, die an 
Literatur.

einen iudex unus ging. Ebenso urteilte ein iudex unus allemal in den Provinzen. Die cognitio extraordinaria war z. B. angebracht, wenn es sich um Restitution eines Erbschaftsfideikommisses handelte (Paul. sent. 4,5,9), oder wenn der Intestaterbe die Érbschaft rom Fiskus herausverlangte (C. 3, 28, 10). In allen diesen Fällen bildete die querela inofficiosi stets ein Inzidentverfahren. Diese Auffassung hat wenigstens das eine Verdienst, daß sie gründlich reinen Tisch macht, und ein einheitliches Erklärungsprinzip für das gesamte Material der schwierigen und vielbestrittenen Frage aufstellt. Sie ist auch auf den ersten Blick sehr bestechend; aber freilich muß sie erst einer eingehenden Prüfung unterworfen werden. Am Schluß der Abhandlung erörtert dann noch Verf. die Bedeutung und Entstehung der fünfjährigen Praescriptio bei der Querel. Sie erklärt sich, wie die praescriptio longi temporis, aus einer Beweisregel (was auch für Verfs. Inzidenthypothese spricht): wer fünf Jahre das Testament nicht anfocht, galt, als habe er es anerkannt. Das Vorbild lieferte die gleichfalls vor den Zentumvirn entwickelte praescriptio gegen denjenigen, der eine Zuwendung aus dem angefochtenen Testamente angenommen hatte. Die Zeitdauer war der Bestimmung Nervas entlehnt, daß fünf Jahre nach dem Tode Jemandes über seinen Status kein Streit mehr erhoben werden dürfe ( $\nabla g l$. äbrigens Mitteis, Zeitschr. d. Sav. Stiftg. XXVII, 227): Die Praxis war es, nicht die Theorie - le palais et non l'école, wie Verf. sagt - welche die querela inofficiosi testamenti schuf und weiter bildete.

In einem feinsinnigen Aufsatze Sur quelques exemples de gémination juridique dans les auteurs littéraires latins behandelt $\mathrm{Gas}$ ton $\mathrm{May}$ die Doppelausdrücke der Juristensprache, die in der nichtjuristischen Literatur vielfach nachgeahmt wurden. Für jura et leges, solvere et liberare gibt er sehr reichhaltige Zusammenstellungen im Anhange, ebenso daselbst eine Liste der Doppelausdrücke im Code civil. Ér erklärt diese auffällige Spracherscheinung aus einer übertriebenen Vorsicht beim Gebrauche juristischer Formeln, die aus der abergläubischen Furcht der bäuerlichen Bevölkerung von Urrom entsprungen sei. Für iura et leges trifft das schwerlich zu, und über die Bedeutung dieser Wendung in der späteren Kaiserzeit hat besonders zutreffend gehandelt Momnsen (Jur. Schr. II, 372f.). Vermißt haben wir in der sonst sehr reichbaltigen Literaturbenutzung einen Hinweis anf Kalbs Juristenlatein, in welchem das hier behandelte Symptom nicht unberücksichtigt geblieben ist. Sehr beachtenswert sind auch die gleichfalls unbenutzten Bemerkungen und Zusammenstellungen von Voigt, XII Tafeln I S. 144. Unbemerkt geblieben ist bisher, so weit ich sehe, die Wendung datio deminutio bei Liv. 39, 19.

Mey nial beschäftigt sich mit der lex Emptorem Cod.4, 66 (65 Krueger), 9, welche dem Käufer eines Grundstückes gestattet, den Pächter, der sein Recht vom Verkäufer herleitet, zu vertreiben. Er verlangt, da $\beta$ dieser Rechtssatz auf Landpacht eingeschränkt und nicht auf Wohnungsmiete ausgedehnt werde. Er zeigt ferner, daß der Erlaß bereits im 4. Jhdt. auf coloni nicht mehr anwendbar war, da diese an die Scholle 
gefesselt waren, von der sie der Dominus nicht vertreiben durfte, daß er also nunmehr nur noch für die conductores, die Generalpächter, in Betracht kam. Indem er dann die dem Erlaß vorangehende Zeit betrachtet, weist er eine Tendenz in der Rechtsentwicklung nach, die dabin geht, die Rechtsstellung des Pächters dem Verpächter oder dessen Singularsukzessor gegenüber zu stärken (wozu freilich Dig. 7, 1, 59, 1 nicht paßt), und gelangt zu dem Resultate, daß unser Erlaß in dieser fortschrittlichen Entwicklung einen Rückschritt bedeutet (une sorte de recul, de pas en arrière), der aber nicht imstande war, den Lauf der Dinge lange aufzuhalten. Verf. sucht das Motiv des reaktionären Gesetzes in dem konservativen Charakter des Alexander Severus, der die unter Elagabals Regierung niedergelegten Schranken $\mathbf{z w i s c h e n}$ den einzelnen Ständen und Klassen wieder aufzurichten bemüht war. In der T'extbehandlung stimme ich Verf. bei, wenn er die Vulgata gegen die handschriftliche Überlieferung herstellen will; für die Datierung des Erlasses ist aber einzig und allein die Subskription maßgebend, wonach er in das Jahr 234, das vorletzte der Regierung des Alexander, gehört. Zu der Frage, ob der zweite Satz interpoliert ist (Pacchioni), nimmt Verf. keine entschiedene Stellung. Konrad Hellwig (Verträge auf Leistung an Dritte S. 36) hat sich, wie wir glauben mit Recht, dagegen ausgesprochen. Dem Verf. sind diese Ausführungen, wie es scheint, nicht bekannt, ebensowenig, wie mein Aufsatz über Sklaven und Kolonen in der Festschrift für Vablen. Ich babe ihn allerdings der allgemeinen Kenntnis einigermaßen entzogen, indem ich ihn in einer Festschrift begraben habe, und ich wünsche dem Aufsatze Meynials ein besseres Los, als es dem meinigen, der völlig unbeachtet geblieben ist, zu teil geworden ist.

In das byzantinische Recht fübrt uns die gelehrte Abhandlung von Monnier, Du casus non existentium liberorum dans les nouvelles de Justinien. Casus heißt zunächst Unfall, Tod, dann aber bezeichnet es auch den Gewinn, der jemandem durch den Tod eines andern erwächst. So sagt Justinian C. 5, 12, 31, 1: nisi ipse, qui ab initio dotem dederit, sibi dari huiusmodi casum stipulatus est. Der xáoos $\dot{\varepsilon} \xi$ áralSias ist der Vorteil, der dem überlebenden kinderlosen Gatten zufällt. Er konnte zunächst nur durch Ehepakten herbeigeführt werden, so auch noch zur Zeit Justinians. Doch hat dieser ihn, da er ibm geeignet schien, die Härte der Strafen der Wiederverheiratung zu mildern, gewissen gesetzlichen Bestimmungen unterworfen, die in einer verlorenen Konstitution am Schlusse des Titels 5, 9 des Kodex standen, und die wiederholt in den Novellen erwähnt werden. Er bestand in einer Quote der Dos oder der Donatio antenuptialis, deren Höhe durch Vertrag festgesetzt wurde. Er konnte nach der Constitutio Leonina v. J. 468 (C. 5, 14, 9) und der Novelle 97, 1, wenn im Ehevertrag eine Eheschenkung ansgesetzt war, nicht einseitig für einen der beiden Gatten ausbedungen werden, sondern nur für beide. Wie die zweite Novelle, die vom Verf. sebr anregend besprochen wird, lehrt, wirkte das pactum orbitatis nur, wenn der überlebende Gatte kinderlos war 
oder sämtliche Kinder vor ihm verstorben waren. So hatte es auch die verlorne Konstitution Justinians bestimmt, deren Restitution durch Cuiacius daher teilwojse falsch ist. Erst in der Novelle 22 bestimmte Justinian, daB der überlebende Gatte, der mehrere Kinder hatte, im Falle des Todes eines oder mehrerer dieser Kinder den casus orbitatis pro rata erhielt. Wie diese Bestimmungen sich mit den Strafen der Wiederverheiratung verquickten, wird vom Verf. klar und anschaulich dargelegt. Sein Thema nötigt ihn zu näherer Betrachtung der Donatio antenuptialis, die, wie er behauptet, zu Jnstinians Zeit eine Gegendos war. Anfgefallen ist uns, daß er dabei von den bekannten Ausführungen in Mitteis' Reichsrecht nicht Notiz genommen hat, obwohl er doch in der deutschen Literatur wohl bewandert ist.

In einer Observatiuncula elegantissime scripta versucht $\mathrm{N}$ a ber in Dig. 47, 10,67, 4-6 und 2, 9,5 ausgedehnte Interpolationen nachzuweisen. Dem Bedenken, das Lenel dagegen in den Nachträgen zur zweiten Ausgabe des Edikts bezüglich der erstgenannten Stelle ausspricht, kann ich mich nur anscbließen. Auch die Interpolation der zweiten Stelle halte ich durchaus nicht für sicher; das Latein ist zwar so schauderhaft, wie Naber es schildert. Ich glaube aber, daß nur die capitales actiones und das supplicium interpoliert sind, daß dagegen Ulpian $\mathrm{zwischen} \mathrm{der} \mathrm{noxalis} \mathrm{actio} \mathrm{iniuriarum} \mathrm{und} \mathrm{den} \mathrm{übrigen}$ actiones noxales einen Unterschied gemacht hatte, der dann freilich durch die Tätigkeit der Kompilatoren verwischt worden ist. Besser wird die Rechtslage des Klägers sicherlich, wenn er gegen den Freigelassenen klagen kann. Denn hat dieser Geld, so erhielt der Kläger vollen Schadensersatz und braucht sich nicht mit der Noxae deditio abspeisen zu lassen. Hat der Freigelassene kein Geld, so führt seine Verurteilung zur Personalexekution (Ductio), und also zu demselben wirtschaftlichen Ergebnis, wie die Noxalklage. Aber wie der Schlusssatz der Stelle jetzt da steht, hat er kein Subjekt, und es muss etwas vorher weggeschnitten sein.

Rabel handelt von der Unmöglichkeit der Leistung im römischen Recht; er will den Ursprung des berühmten Satzes 'impossibilium nulla obligatio' ergründen. Ungültig war bei den Römern eine Stipulation, die auf Leistung einer nicht existierenden Sache (quae in rerum natura non est) gerichtet war (physische Unmöglichkeit); ebenso das Damnationslegat und der Kauf, wenn die vermachte oder verkaufte Sache nicht existierte. Diese Sätze sind sehr alt; sie stammen aus republikanischer Zeit (veteres). Wabrscheinlich ebenso alt ist der Satz, daß Stipulation, Legat, Kauf einer dem Commercium entzogenen Sache nichtig ist (juristische Unmöglichkeit); nur bezüglich des fundus hostium und des in feindlicher Gewalt befindlichen Sklaven bestand Meinungsdifferenz, indem einige Kanf (Vermächtnis) einer solchen Sache nicht für ungültig, sondern nur für aufschiebend bedingt hielten, und Octavenus das Commercium 2 wischen den Kontrahenten schied vom allgemeinen Commercium. Bei beiden Kategorien der Unmöglichkeit, der physischen wie der juristischen, kam nur die Beschaffenheit der zu 
leistenden Sache in Betracht, nicht die Handlung (der Erfüllungsakt, datio). Diese wurde erst später herangezogen: natura fieri non concedit, Paul. Dig. 45, 1, 35 pr. Um auszudrïcken, ob eine Handlung ihrer Natur nach möglich oder unmöglich sei, verwandten dann die Juristen die Adjectiva; possibilis und impossibilis. Wie alt sind sie? Verf. handelt darüber ebenso eingehend und sorgfältig, wie vorher über natura rerum. Labeo kannte das Wort impossibilis nicht; er sagte dafür ảdv́varos. Aber wenn Scialoja den Gebrauch der Adiectiva possibilis und impossibilis allen Juristen vor Julian abspricht, so will Rabel nicht so weit herabgehen; er schreibt ibre Einführung in die Rechtssprache dem Sabinus zu. Seine beiden Beweisstellen sind jedoch bedenklich. Dig. 45, 1, 137, 5 (Venul.), cum alio possibile sit, iure factam obligationem Sabinus scribit, möchte ich den falschen Dativ 'alio' nicht dein Sabinus zutrauen (Voc. Iur. Rom. I 366, 25; Ulp. Dig. 36, 1, 17 (16), 12 ist interpoliert: bei Paul. Dig. 2, 14, 28, 2 ist alio Ablativ; vgl. Berl. Philol. Wochenschr. 1908, Sp. 530f.); er könnte geschrieben haben: cum alius facere potest. An der andern Stelle, D. 35, 1, 72, 7 liegt Referat von Papinian vor, das schwerlich die Ansichten von Cassius und Caellius Sabinus im Wortlaut wiedergibt. Doch dürften die in Frage stehenden Ausdrücke, die wir bei Gaius bereits fest eingebürgert finden, schon in ersten christlicben Jahrhundert in die juristische Literatur eingedrungen sein. Wie steht es nun mit der Abstammung der Begriffe? sind sie ans der Philosophie in die Jurisprudenz übernommen? Bei der Untersuchung dieser Frage hat Verf. wieder Gelegenheit, mit seiner profunden Kenntnis der antiken Literatur zu glänzen. Die Ergebnisse seiner Studien weichen ziemlich stark ab von denen Sokolowski's. So sehr die gebildeten Römer auch mit der philosophischen Literatur vertraut waren, so entnahmen sie ibr doch weder Begriffe noch Sätze für das Recht, oder wenn sie das wirklich getan haben sollten, so haben sie dieselben für ihre Zwecke hergerichtet. Für die praktischen Bedürfnisse des Rechts waren die abstrakten Theorien der Philosophie nicht ohne weiteres brauchbar. Dieser gewiß richtige Gedanke findet seine Bestätigung in Cicero's und Seneca's philosophischer Schriftstellerei. Verf. ninmt daher an, daß der Satz von der Ungültigkeit der Rechtsgeschäfte, soweit es sich um nicht existierende Sachen handelt, aus dem ältesten Rechte der Römer stammt, welches sich nur mit körperlichen, sinnlich wahrnehmbaren Sachen befaßte. Er gibt dann noch einen raschen Überblick über die weitere Entwickelung und Ausdehnung der alten Rechtsregel, die Scheidung 2 wischen absoluter und relativer, objektiver und subjektiver Unmöglichkeit, wobei er geneigter ist, einen Einfluß der Philosophie anzunehmen; man ließ Ausnahmen zu und erklärte den Kauf fremder Sachen, ja selbst von res extra commercium für gültig. Die nachträgliche Unmöglichkeit ist im älteren Recht niemals ein Grund für die Nichtigkeit der Obligation gewesen; sie hat auch in der Theorie der contractus bonae fidei nur eine nebensächliche Rolle gespielt.

Der letzte Aufsatz der schönen Sammlung 'Les piae causae dans le droit de Justinien' von Saleilles gespendet, führt uns noch einmal 
in das justinianische Recht. Verf. knüpft seine Betrachtungen an einen Aufsatz von Stanislao Cugia in der Festschrift für Fadda, in den nachgewiesen war, daß der Ausdruck 'pia causa' zu Justinians Zeit lediglich den frommen Zweck, das Motiv der Liberalität, nicbt das fromme Werk selbst, die Stiftung bezeichnete. Die Richtigkeit dieses Satzes vorausgesetzt, entfällt ein schwerwiegendes Argument für die Existenz von Stiftungen im römischen Recht. Die Nichtexistenz der Stiftungen ist aber damit keineswegs bewiesen. $\mathrm{Ob}$ die mildtätigen Anstalten, deren Rechtsverhältnisse im Justinianischen Corpus geregelt werden, Stiftungen sind oder nicht, das kann nur aus den Quellen und aus den Tatsachen entschieden werden. Schon der Ausdruck selbst zeigt, daß mit den piae causae oder $\varepsilon \dot{v} \sigma \varepsilon \beta \varepsilon \tilde{\varepsilon} s$ aitial doch nicht immer nur das Motiv, sondern bisweilen auch schon das Resultat, die Stiftung selbst, gemeint ist, so wenn es Cod. I, 3, 45, 1a heißt: $\varepsilon i$ d $\dot{\varepsilon}^{\text {a }} \tau \iota \nu \alpha$

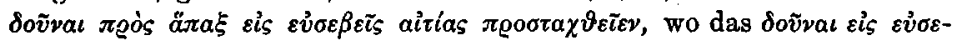
Beĩs aitias (dare in pias causas) auf eine Linie zu stellen ist mit den donationes ... in sanctam ecclesiam vel in xenodochium vel in nosocomium C. 1, 2, 19. Aber ganz abgesehen von dem Ausdruck beweist die Organisation, die Selbständigkeit der Verwaltung, die Vermächtnisfähigkeit dieser Werke der Barmherzigkeit ihren selbständigen Charakter und kennzeichnet sie als wirkliche Stiftungen mit eigner juristischer Persönlichkeit. Die Zuwendungen werden nicht der Kirche unter Auflage gemacht, sondern entweder einer bereits bestehenden Stiftung (Waisen-

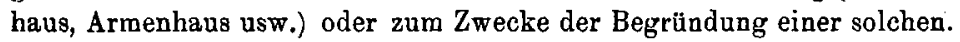
Die Zugehörigkeit der Kirche zeigt sich nur in dem Aufsichtsrecht der Kirche und darin, daß Stiftungen nur zu charitativen $\mathrm{Zwecken} \mathrm{(pieta-}$ tis intuitu C. 1, 3, 48 pr.) zugelassen sind. Verf. schließt sich hier ganz der Ansicht Sohm's an, die in dessen Institutionen $\$ 38$ vorzüglich formuliert ist. Demnach sieht er in den Piae causae keine Stiftungen im modernen Sinn, keine Personifikation des Zwecks, kein Zweckvermögen; vielmehr hält er für die Vermögensträger der gestifteten Summen die Gesamtheit derer, zu deren Gunsten die 7uwendung bestimmt ist, die Armen, Kranken usw. Er findet in den Finrichtungen der Mildtätigkeit ein korporatives Element. Einer Genehmigung seitens der Regierung bedurften die Piae causa zur Erlangung der Rechtsfähigkeit nicht. Sie wurde begründet durch den Willen des Stifters, dem, sofern er auf einen frommen Zweck gerichtet war, vom Gesetze die Rechtswirkung zuerkannt wurde. Die Konsekration der Stiftung durch den Priester hatte nur religiöse, keine juristische Bedeutung; sie wurde auch bloß bei Kirchen, Bethäusern und Klöstern angewandt, nicht bei den übrigen Anstalten der Barmherzigkeit. 'Nicht das System der Verleihung der Persönlichkeit, noch weniger das der fiktiven Persönlichkeit erscheint in unsern Texten. Die Stiftung repräsentiert wirkliche Personen; das Vermögen gehört wirklichen Personen und wird in autonomer Form verwaltet, mit der Maßgabe, daß die Einkünfte zu.dem rom Stifter bestimmten $Z_{w}$ eck verwandt werden.' Diese rechtliche Gestaltung der Stiftung ist nahe verwandt derjenigen, welche 
die Stiftung im beutigen englischen Recht hat; die römischen Verwalter der Stiftungen sind den englischen trustees, insonderheit den charitable trustees sehr ähnlich. Verf. hat romanistisch begonnen und schließt germanistisch. Das bedeutet aber nicht etwa, daß seine Ideen unsicher oder unlogisch wären. Im Gegenteil! Seine Auffassung ist nicht nur nach jeder Richtung hin durchdacht, sondern auch durchaus konsequent und geschlossen. Allerdings bedeutet sie eine Vermittelung zwischen den beiden Extremen; aber es wäre ja hier nicht das erste Mal, daß die Wahrheit in der Mitte liegt.

Der schöne Band, den wir betrachtet baben, beginnt wit einer Frage des ältesten, er schließt mit einem Gegenstand des spätesten römischen Rechts. Von der Reichbaltigkeit und Gediegenheit des Inhalts hoffen wir durch unsere Übersicht dem Leser einen Begriff verschafft gu haben. $\mathrm{Da} \beta$ den Beschlu $B$ gerade eine $A$ bhandlung über die piae causae bildet, ist abermals ein sinnreicher und nicht bedeutungsloser Zufall. Denn der ganze Band ist ein Akt der Pietät; und wenn 2weiundzwanzig hervorragende Juristen ihre Arbeit in den Dienst der Liebe und Dankbarkeit gegen einen verehrten Meister stellen, so ist das ein schönes Zeugnis für die ideale Gesinnung, mit der sie ibres hohen Priesteramtes walten.

B. Kübler.

Zoc co-Rosa, Iustiniani Institutionum Palingenesia. (Annuario dello Istituto di Storia del Diritto Romano, Vol. IX, p. 180-439, Vol. X, p. 1-227, Catania 1905-1908).

Die Quellenanalyse der Institutionen Justinians, welche der Prăsident des rechtshistorischen Instituts von Catania dem gelehrten Publikum unterbreitet, umfaßt bisher nur die beiden ersten Bücher. Sie ist mit rühmlicher Vorsicht und musterhafter Grïndlichkeit geführt. Alle Vorarbeiten sind gewissenhaft berücksichtigt, aber auch gebührend kritisiert. Am schärfsten werden die Arbeiten von Ferrini geprüft, über die ich im XXIII. Bande dieser Zeitschrift S. 508-526 ausführlich berichtet habe. Mein Urteil stimmt im wesentlichen überein mit dem ron ZoccoRosa, welcher hier die Aufgabe leistet, die ich am Schlnß meines Referats verlangte, nämlicb eine Nachprüfung der Untersuchung Ferrinis Paragraph für Paragraph und eine Angabe der mutmaßlichen Quellen der Institutionen unter kurzer Angabe der Gründe, Satz für Satz. Während Ferrini, indem er seiner Phantasie allzusehr die Zügel schießen ließ, oftmals ohne hinreichende Gründe Vermutungen über die Quellen einzelner Abschnitte aufstellte und dann obendrein die Hypothesen von den sicheren Tatsachen nicht schied, führt Zocco-Rosa dies alles auf das richtige $\mathrm{MaB}$ zurück. Er verlangt, daß niemals eine Quelle als sicher bezeichnet wird, die nicht mit voller Bestimmtheit, insonderheit durch Vergleichung mit anderweitig überlieferten Texten, als solche erkannt ist, sondern nur durch gewisse Indizien des Stiles oder 
des Inhaltes ermittelt ist. Er fordert ferner, daß auch bei solchen Stellen, deren Quelle sicher erkannt ist, die Veränderungen, welche die Kompilatoren mit ihrer Vorlage vorgenommen haben, angedeutet werden. Da häufig ein Paragraph aus nehreren Vorlagen zusammengesetzt ist, so bleibt er nicht bei der Paragrapheneinteilung stehen, sondern zerlegt - und dieses Verfabren verdient ganz besondere Anerkennung - die Paragraphen, wo es nötig ist, wieder in mehrere Abschnitte.

Es ist kaum zu erwarten, daß wir bei dem derzeitigen Bestande unserer Überlieferung über die von Zocco-Rosa festgelegten Resultate noch weit hinauskommen werden. Die Mittelchen, welche Ferrini als zur Auffindung von Quellen geeignet entdeckt zu haben vermeinte, hat Z. R. einer vernichtenden Kritik unterzogen; er denkt darüber nicht anders, als ich. Nur das sprachliche Kriterium, das ich schon früher als das brauchbarste von allen erklärte, halte ich immer noch für besser, als es $Z$. R. hinstellt, der es gründlich verachtet. Zwar daß es vielfach gemißbraucht wird, daß es namentlich von Ferrini oft ganz verkehrt angewandt worden ist, soll keinen Augenblick bestritten werden. Es war verfehlt, wenn Ferrini für Inst. 2, 4, 2 goianischen Ursprung annahm, weil dort steht: exceptis his, quae und die gleiche Wendung Gai. III, 83 begegnet; denn sie findet sich auch bei Paulus Dig. 50, 7, 11 (10) pr.; Sent. III, 6, 44; 69 und bei Hermognoian Dig. 27, $1,41,3$ und auch sonst; nicht minder verkehrt war es, Inst. 2, 12, 1 dem Gaius zuzuweisen auf Grund der Wendung: nec ad rem pertinet, die in V. I. R. I p. 148, 36 nicht weniger als 46 mal aus allen möglichen Juristen belegt ist, oder Inst. 2, 11, 3 dem Marcian wegen des Ausdrucks hactenus-quatenus, der $25 \mathrm{mal}$ in unsern Quellen vorkonmt, aber gerade beim Marcian niemals. Aber ist ein Instrument darum schlecht, weil es nicht jeder zu spielen versteht? Im Gegenteil, je feiner es ist, einen desto größeren Künstler verlangt es. Ich habe schon in meiner Besprechung der Untersuchung Ferrinis diesem den Vorwurf gemacht, daß er 'das Vocabularium Iurispr. Rom. nicht gebraucht hat; ich hätte Grund, ihn Z. R. gegenüber zu wiederholen. Wozu wird eine unsägliche Mühe und Arbeit auf dies Werk verwendet, wenn es nicht einmal für solche Untersuchungen, wie die vorliegende, benutzt wird? Un die völlige Wertlosigkeit der sprachlichen Indizien zu zeigen, kommt Z. R. immer wieder - er ist überhaupt den Wiederholungen nicht abgeneigt - auf Inst. 1, 1, 2 zurück. Diesen Paragraphen erklärte Ferrini zuerst für tribonianisch; dann nahm Kalb ihn als klassisch in Anspruch und wies ihn vermutungsweise dem Marcian zu. Ferrini stimmte in seiner zweiten Untersuchung Kalb in bezug auf den klassischen Ursprung der Stelle bei, vermutete aber als Quelle Gains. Diese letztere Annahme suchte ich in meiner Anzeige (diese Zeitschr. XXIII, 511) durch mehrere Argumente sprachlicher Art zu stützen, unter Zustimmung von Kalb. Über einen solchen Wechsel von Ansichten gießt nun Z. R. an vielen stellen seiner Untersuchung die volle Schale seines Hohns aus; die sprachlichen Kriterien, sagt er, führen also bald dazu, eine Stelle dem 
Tribonian, bald dem Marcian, balil dem Gaius zuzuweisen! Wie unberechtigt ist indessen ein solcher Hohn! Die Geschichte dieses Paragraphen zeigt weiter nichts, als wis in der Wissenschaft ganz gewöhnlich ist, daB die Erkenntnis der Wabrheit sich nur allmählich Bahn bricht, zumal wenn sie, wie hier, zunächst mit unzulänglichen Mitteln gesucht wird. Der Hauptbeweis dafür, daß der Paragraph 2 dem Gaius entnommen ist, liegt in der Wendung statim ab initio, wie ich in dieser Zeitschr. XXIII S. 511 und in der Praefatio zum Gaius p. IV n. 1 ausgeführt habe. Dazu bemerkt Z. R. (Tom. IX p. 384): daß Gaius öfters die Wendung 'statim ab initio' gebraucht, batte schon i. J. 1824 Christian Friedrich Elvers in seinem Promptuarium Gaianum bemerkt, aber ohne Ūbertreibungen (senz' esagerazioni). Was diese verletzende Bemerkung soll, weiß ich nicht. In einem Promptuarium Gaianum zählt man eben die Stellen des Gaius auf; Utbertreibungen kann man da gar nicht anbringen. Sie sind auch im Voc. Iurispr. Rom. nicht angebracht. Habe ich denn aber überhaupt ïbertrieben? Ist es denn etwa nicht wahr, was ich in dieser Zeitschrift und in der Praefatio zum Gaius schrieb, daß die Wendung 'statim ab initio' eben nur bei Gaius sich findet? Und ist es nicht ebenso wahr - was $Z$. R. wohlweißlich verschweigt - daß, wie sich aus V.J.R.I p. 56, 41f. ergibt, ab initio in unsern Quellen $100 \mathrm{mal}$, sage und schreibe $100 \mathrm{mal}$, begegnet, statim $a b$ initio aber nur beim Gaius und zwar nicht weniger als 12 mal (nämlich Dig. 35, 2, 73, 5; 35, 2, 76, 1;35, 2, 78;46, 1, 70, 1 (zweimal). Inst. II, 123 (zweimal). 146 (ergänzt aus lnst. $2,17,5$ (4). 148. IV, 64 (nach Huschkes Coniectur). 172. 173) oder wenn man IV, 64 wegläßt, worauf wenig ankommt, $11 \mathrm{mal}$. Kann man demgegenüber noch von Zufal] reden? Oder ist es eine Übertreibung, wenn die Wendung 'statim $a b$ initio' als dem Gaius eigentümlich bezeichnet wird? Oder hat es etwa die allergeringste Bedeutung, ảß sie, wie Z. R. hervorhebt, sich im ersten und dritten Buche der Institutionen des Gaius nicht findet? Natürlich ist die Möglichkeit nicht völlig ausgeschlossen, daß sich diese Wendung in den verloraen Institutionen des Florentinus, Ulpianus, Marcianus fand; aber bei Ulpian ist die Wabrscheinlichkeit sehr gering, da er laut Vocabularium an 47 erbaltenen Stellen seiner Werke ' $a b$ initio', niemals 'statim ab initio' schrieb, und bei Florentinus und Marcianus, bei denen ' $a b$ initio' allerdings nicht belegt ist, ist doch nicht gerade anzunelzmen, daß sie sich anders ausgedrückt haben sollten, als die M!ehrzahl der klassischen Juristen erweislich getan hat. Geleugnet we1den soll auch nicht die Möglichkeit, da $\beta$ die Kompilatoren, die ja ihren Stil nach dem Muster der klassischen Juristen bildeten und gera de den Gaius manchen Ausdruck entlehnt haben, ihm auch das charakteristische 'station ab initio' entnahmen. Nachdem aber der in Frage sitehende Paragraph seiner ganzen Ausdrucksweise nach als klassisch erlannt ist, so sind wir wohl berechtigt, wenn wir in ihm eine Lieblingswendung des Gaius finden, ihn mit einiger Wahrscheinlichkeit diesem Juristen zuzuweisen, zumal sich in ihm noch mehrere andere für Ga ius charakteristische Ausdrücke 
finden, vor allem das ganz eigentümliche duorum alterum aut-aut. Es ist also Z. R. nur das eine zuzugeben, daß man nicht von absoluter Sicherheit, sondern nur von einem hohen Grade der Wahrscheinlichkeit sprechen sollte. Mehr besagt auch, streng genommen, das 'certe' nicht, dessen ich mich in der Praefatio zum Gaius bediente, das ich aber in Zukunft, um jedes Mißverständnis auszuschließen, durch einen vorsichtigeren Ausdruck ergetzen werde.

Um den Stil eines Schriftstellers zu erkennen, bedarf es sehr sorgfältiger Beobachtung und feinen Empfindens. Wem diese Gaben nicht von der Natur verliehen sind, der wird sie nie erlernen; aber auch beim Begabten mússen sie erst durch lange Ưbung gebildet und entwickelt werden. Das gilt vom Stilgefühl der Kunstprosa gegenüber gerade ebenso, wie gegenüber der Plastik, Malerei, Musik. Mathematische oder, wie sie Z. R. nennt, objektive Beweise, lassen sich hier nicht liefern; aber Resultate lassen sich deshalb doch gewinnen. Die Literaturgeschichte gibt Beispiele genug dafür. Notwendig ist es freilich, die Beobachtung auf das kleinste zu richten, eine gleichsam mikroskopische Untersuchung anzustellen, und das will auch gelernt sein. Ferrini behauptete für Inst. 2, 17 pr. gajaniscben Ursprung, weil sich hier eo usque donec findet, was, wie Kalb (Roms Juristen S. 75/76) gezeigt habe, nur bei Gaius vorkomme. Demgegenüber behauptet Z. R. (Vol. X p. 130) diese Wendung finde sich auch bei Marcian Dig. 20, 1, 11, 1 (worauf schon Ferrini hinweist), nur mit der leichten Unstellung usque eo, und bei Pomponius Dig. 41, 2, 25, 2. Vor mir liegt der Artikel 'donec', den Grupe für das Vocabularium angefertigt hat. Durch ihn wird die Beobachtung Kalbs, wie fast immer, voll und ganz bestätigt. Weder Ferrini noch Z. R. haben aber gesehen, worauf es ankommt. Beide haben Kalb falsch zitiert. Die Stellung usque eo oder eo usque ist viel weniger maßgebend, als die Bedeutung. Was sagt denn Kalb in Wirklichkeit? Daß eo usque donec in der auffallenden Bedeutung "so lange als' nur bei Gaius gefunden wird, und das ist richtig. In der Bedeutung "so lange bis' findet sich die Wendung auch bei Julian, Pomponius und Marcian. Das Material verteilt sich folgendermaßen. eo usque, donec $=$ so lange als: Gai. Dig. $23,2,17$ pr.; 41, 1, 2, 2; 41, 1, 5,$1 ; 4 ; 5$. Inst. II, 67. III, 151. - eo usque donec $=$ bis : Jul. Dig. 40, 12, 30. Pomp. Dig. 1, 2, 2, 51. Gai. Dig. 9, 4, 27 pr. Inst. II, 213. III, 184 (zweimal). Marcian. Dig. 20, 1, 11, 1 (auch hier eo usque, nicht, wie Z. R. behauptet, usque eo). - usque eo donec = bis Pomp. 41, 2, 25, 2 (zweimal). Wie steht es nun aber mit der Institutionenstelle? Hier ist usque eo donec in der Bedeutung 'bis' gesetzt:

Testamentum iure factum usque eo valet, donec rumpatur irritumve fiat.

Mit Unrecht berief sich also Ferrini auf Kalb, dessen Ausführungen er nicht verstanden hatte. Das Indizium, das er für den gajanischen Ursprung der Institutionenstelle vorbrachte, ist falsch. In so weit hat Zocco-Rosa recht; den wahren Grund ron Ferrinis Irrtum hat aber auch er nicht erkannt. Diese Bemerkung mag zeigen, wie behutsam 
solche Untersuchungen zu führen sind. Zocco-Rosa verdient freilich Zustimmung, wenn er immer wieder zur Vorsicht mahnt und namentlich Einspruch dagegen erhebt, daß Vermutungen für Tatsachen ausgegeben werden; aber mit der reinen Negative gegenaber allen Sprachbeobachtungen ist es auch nicht getan, und das schroffe Ablebnen oder Verböhnen jeder Vermutung, mag sie geistreich sein oder nicht, ist zwar sehr bequem, trägt aber auch zur Vermehrung unserer Einsicht nicht viel bei.

\section{B, Kübler.}

Kretschmar, Paul, Die Erfüllung. Erster Teil. Historische und dogmatische Grundlagen. Leipzig 1906. Verlag von Veit \& Co. $8^{\circ}$ und 168 S. ${ }^{1}$ )

Es ist gewiß ein richtiger Gedanke, der Lehre von der Obligationserfüllung größere Aufmerksamkeit zuzuwenden, als bisher geschehen ist; denn die Erfüllung gibt überall den MaBstab für den Inhalt des Forderungsrechts und darum ist nicht $z u$ verkennen, da $B$ man von den für sie geltenden Rechtssätzen aus sich vielfach über die Struktur des Forderungsinbalts selbst informieren kann. Uber die Fragen, welche hierbei in Betracht kommen können, orientiert man sich teilweise aus dem ${ }_{n} z$ weiten Teil ${ }^{\mu}$ des vorliegenden ersteo Bands, welcher in das Dogmatische der Lebre ubergeht und, wie es scheint, als allgemeiner Teil der Erfüllungslehre gedacht ist, dem spezielle Betrachtungen noch folgen sollen. Insbesondere aus den $\$ 86,7$ und 9 ist Einblick in die Probleme zu gewinnen: Ist die Erfüllung Rechtsgeschäft - etwa sogar Vertrag - oder Tathandlung? Eine andere Frage ist die, ob jede Befriedigung des Obligationszwecks Erfüllung darstellt oder nur die Verwirklichung des spezifischen Obligationsinhalts, ob also der Concursus cansarum Incrativarum der Erfüllnng beizuzählen, ob die Leistung durch einen Dritten wahre Erfullung ist; auch danach läßt sich fragen, ob die Zwangsbeitreibung unter den Begriff fallt, was z. B. von Siber (Rechtszwang in Schuldverhaltnisien 8. 154f.) bejaht, vom Verf. verneint wird. Noch andere Probleme setzen sich an; das Thema laBt sich weit ausspinnen. Allerdings ist dasselbe auch keineswegs ungefährlich. Da man bei der Frage nach der richtigen Erfüllung immer auf das eigentliche Wesen des Forderungsrechts zurückgefuhrt wird, kommt man leicht ins Ungemessene und betritt man oft schwankenden Boden; es ist äußerst schwierig, hier lauter zu Ende gedachte Gedanken zu geben und fast unmöglich, die Summe der einschlagenden Fragen nu tbersehen und zu ordnen. Wie weit dem Verf. dies gelungen ist, wird man denn auch erst nach dem Erscheinen des zweiten Bandes sagen können; der vorliegende

1) Die Anzeige dieser Schrift des Verfs. sowie anch seiner Arbeit über die "Entwicklung der Kompensation" (S. 457) geachieht ohne Schuld der Redaktion verspatet, weil die Einsendung zur Rezension infolge eines Mißverstkndnisses erst hever erfolgt ist. 